\title{
Simultaneous multicolour optical and near-IR transit photometry of GJ 1214b with SOFIA ${ }^{\star}$
}

\author{
D. Angerhausen ${ }^{1,13,14, \star \star}$, C. Dreyer ${ }^{2}$, B. Placek ${ }^{3}$, Sz. Csizmadia ${ }^{2}$, Ph. Eigmüller $^{2}$, M. Godolt $^{2}, 11$, D. Kitzmann ${ }^{1}$, \\ M. Mallonn ${ }^{8}$, E. E. Becklin ${ }^{5,9}$, P. Collins ${ }^{4, \star \star \star}$, E. W. Dunham ${ }^{4}$, J. L. Grenfell ${ }^{2}$, R. T. Hamilton ${ }^{9}$, P. Kabath ${ }^{6}$, \\ S. E. Logsdon ${ }^{5}$, A. Mandell ${ }^{13}$, G. Mandushev ${ }^{4}$, M. McElwain ${ }^{13}$, I. S. McLean ${ }^{5}$, E. Pfueller ${ }^{7}$, H. Rauer ${ }^{2,11}$, \\ M. Savage ${ }^{12}$, S. Shenoy ${ }^{9}$, W. D. Vacca ${ }^{9}$, J. E. Van Cleve $^{10}$, M. Wiedemann ${ }^{7}$, and J. Wolf ${ }^{7}$ \\ ${ }^{1}$ Center for Space and Habitability, University of Bern, Sidlerstrasse 5, 3012 Bern, Switzerland \\ e-mail: daniel . angerhausen@csh.unibe.ch \\ 2 Department of Extrasolar Planets and Atmospheres, Institute of Planetary Research, German Aerospace Center, \\ Rutherfordstrasse 2, 12489 Berlin, Germany \\ 3 Department of Sciences, Wentworth Institute of Technology, Boston, MA 02115, USA \\ ${ }^{4}$ Lowell Observatory, 1400 West Mars Hill Road, Flagstaff, AZ 86001, USA \\ 5 Department of Physics and Astronomy, University of California Los Angeles (UCLA), 465 Portola Plaza, Los Angeles, CA 90095, \\ USA \\ 6 Astronomical Institute ASCR, Fričova 298, 25165 Ondrějov, Czech Republic \\ 7 Deutsches SOFIA Institut, University of Stuttgart Pfaffenwaldring 29, 70569 Stuttgart, Germany \\ 8 Leibniz-Institut für Astrophysik Potsdam (AIP), An der Sternwarte 16, 14482 Potsdam, Germany \\ 9 USRA-SOFIA Science Center, NASA Ames Research Center, Moffett Field, CA 94035, USA \\ 10 SETI Institute, 1533 16th Place, Longmont, Colorado 80501, USA \\ 11 Centre for Astronomy and Astrophysics, TU Berlin, Hardenbergstrasse 36, 10623 Berlin, Germany \\ 12 University of California Observatories, University of California, Santa Cruz, 1156 High Street, Santa Cruz, CA 95064, USA \\ 13 Exoplanets and Stellar Astrophysics Laboratory, Code 667, NASA Goddard Space Flight Center Greenbelt, MD 20771, USA \\ 14 Blue Marble Space Institute of Science, 1001 4th ave, Suite 3201 Seattle, Washington 98154, USA
}

Received 2 April 2017 / Accepted 22 July 2017

\begin{abstract}
Context. The benchmark exoplanet GJ $1214 \mathrm{~b}$ is one of the best studied transiting planets in the transition zone between rocky Earthsized planets and gas or ice giants. This class of super-Earth or mini-Neptune planets is unknown in our solar system, yet is one of the most frequently detected classes of exoplanets. Understanding the transition from rocky to gaseous planets is a crucial step in the exploration of extrasolar planetary systems, in particular with regard to the potential habitability of this class of planets.

Aims. GJ 1214b has already been studied in detail from various platforms at many different wavelengths. Our airborne observations with the Stratospheric Observatory for Infrared Astronomy (SOFIA) add information in the Paschen- $\alpha$ cont. $1.9 \mu \mathrm{m}$ infrared wavelength band, which is not accessible by any other current ground- or space-based instrument due to telluric absorption or limited spectral coverage.

Methods. We used FLIPO, the combination of the High-speed Imaging Photometer for Occultations (HIPO) and the First Light Infrared TEst CAMera (FLITECAM) and the Focal Plane Imager (FPI+) on SOFIA to comprehensively analyse the transmission signal of the possible water-world GJ $1214 \mathrm{~b}$ through photometric observations during transit in three optical and one infrared channels. Results. We present four simultaneous light curves and corresponding transit depths in three optical and one infrared channel, which we compare to previous observations and current synthetic atmospheric models of GJ 1214b. The final precision in transit depth is between 1.5 and 2.5 times the theoretical photon noise limit, not sensitive enough to constrain the theoretical models any better than previous observations. This is the first exoplanet observation with SOFIA that uses its full set of instruments available to exoplanet spectrophotometry. Therefore we use these results to evaluate SOFIA's potential in this field and suggest future improvements.
\end{abstract}

Key words. planets and satellites: individual: GJ 1214b - planets and satellites: atmospheres - techniques: photometric methods: observational - methods: data analysis

\section{Introduction}

\subsection{GJ $1214 b$}

Since the detection of the transiting super-Earth GJ $1214 \mathrm{~b}$ its true nature has been the subject of great interest and is still

\footnotetext{
* Tables of the lightcurve data are only available at the CDS via anonymous ftp to cdsarc.u-strasbg. fr (130.79.128.5) or via http://cdsarc.u-strasbg.fr/viz-bin/qcat?]/A+A/608/A120 $\star \star$ USRA-NASA postdoctoral fellow.

$\star \star \star$ Deceased, January 16, 2017.
}

strongly debated. Discovered within the MEarth programme (Nutzman \& Charbonneau 2008) by Charbonneau et al. (2009), GJ 1214 b has a radius only 2.7 times larger, while its mass is 6.5 times that of Earth. It transits a nearby $(12.95 \pm 0.9 \mathrm{pc})$ M4.5V star with an orbital period of 1.5804 days and has a semi-major axis of 0.0197 AU (Harpsøe et al. 2013). This results in a planet-to-star flux ratio comparable to that of a Jupiter-sized planet orbiting the Sun, which makes it one of the few super-Earth atmospheres that can currently be investigated with transit spectroscopy. Previous mass and radius measurements of GJ $1214 \mathrm{~b}$ can be explained by various 
interior structure and composition models, for example with large or small water inventories, depending on the assumptions made for the planetary atmosphere (as e.g. described by Rogers \& Seager 2010; Nettelmann et al. 2011). Differentiating between the various water or hydrogen-dominated atmospheres could help distinguish between these interior and composition scenarios and would help set constraints on the formation history of this planet, which has no counterpart in the solar system. This degeneracy can be broken, and the composition of the planetary atmosphere constrained, by observing transmission spectra of the planet's atmosphere. Such measurements have been performed for GJ 1214 b by several groups using either space telescopes such as the Hubble Space Telescope (HST; Berta et al. 2011; Kreidberg et al. 2014) and Spitzer (Désert et al. 2011; Fraine et al. 2013) or ground-based facilities such as Very Large Telescope (Bean et al. 2010, 2011), Canada France Hawaii Telescope (Croll et al. 2011), Gran Telescopio Canarias (Murgas et al. 2012; Wilson et al. 2014), InfraRed Survey Facility (Narita et al. 2013b), and Large Binocular Telescope (Nascimbeni et al. 2015). First observations suggest a flat transmission spectrum at short wavelengths for GJ $1214 \mathrm{~b}$ (Bean et al. 2010), which is consistent with an atmosphere composed of at least $70 \% \mathrm{H}_{2} \mathrm{O}$ by volume. An alternative interpretation of the data is that GJ 1214b's atmosphere is hydrogen-dominated. In this case high-altitude clouds or hazes diminish molecular absorption features at short wavelengths more effectively than at longer wavelengths, whereas a water-rich atmosphere would produce a flat spectrum across all wavelengths. Some measurements support the featureless spectrum (Crossfield et al. 2011; Désert et al. 2011), whereas other observations indicate large features around the $g$ band and the $K$ band, which would imply a $\mathrm{H}_{2}$-rich atmosphere (Croll et al. 2011; de Mooij et al. 2012, 2013; Teske et al. 2013).

The emerging class of super-Earths or mini-Neptunes is likely to be common in the Galaxy (e.g. Marcy et al. 2014). Furthermore, these planets represent an important stepping stone in the data-driven pathway towards characterising Earth-like exoplanets. GJ 1214b has been the subject of many atmosphere modelling studies, focusing on for example the impacts of clouds or chemistry on the spectral appearance and characterisation of the planet (Miller-Ricci \& Fortney 2010; Miller-Ricci Kempton et al. 2012; Howe \& Burrows 2012; Menou 2012; Benneke \& Seager 2013; Morley et al. 2013; Hu \& Seager 2014). Detailed studies considering the formation of clouds and atmospheric dynamics that aim to investigate the formation and nature of clouds and hazes in the atmosphere of GJ 1214b include, for example, Morley et al. (2015) or Charnay et al. (2015).

In order to distinguish between a water-dominated atmosphere (larger mean molecular weight, i.e. smaller scale height) and a hydrogen-dominated atmosphere (smaller mean molecular weight, i.e. larger scale height), we performed a SOFIA primary transit observation of GJ $1214 \mathrm{~b}$. This transit observation specifically targeted the water band around $1.85 \mu \mathrm{m}$ using FLITECAM's extremely narrow-band Paschen- $\alpha$ cont. filter centred at $1.90 \mu \mathrm{m}$ (designed to target the Paschen- $\alpha$ continuum). This wavelength is especially interesting since different results have been observed in the $K$ band around $2.2 \mu \mathrm{m}$ and our Paschen- $\alpha$ cont. data point adds another important adjacent data point close to the $K$ band.

\subsection{Observing exoplanets with SOFIA}

A measurement close to the $1.85 \mu \mathrm{m}$ water band is only possible with SOFIA: telluric absorption almost completely shuts down this band between $H$ and $K$ from ground-based observatories and available space-based telescopes do not cover that wavelength regime. Furthermore, SOFIA provides the only platform for simultaneous optical and infrared observations that are inaccessible from the ground. When conducted from ground-based platforms, spectrophotometric exoplanet observations are significantly affected by the perturbing variations of trace gases, in particular $\mathrm{H}_{2} \mathrm{O}$, in the Earth's atmosphere. It was theorised that the airborne platform SOFIA had some unique advantages for this kind of exoplanet research (Dunham et al. 2007; Gehrz et al. 2010; Angerhausen et al. 2010, 2014; Cowan et al. 2015). SOFIA's cycle 1 observation of HD 189733 b then demonstrated that SOFIA can overcome the hurdle of changing atmospheric absorption in the optical in absolute photometry without the use of field stars; Angerhausen et al. (2015) demonstrated a precision of $\sim 150 \mathrm{ppm}$ in absolute optical photometry of HD 189733b. SOFIA can leverage bright host stars to the fullest and is therefore not limited in $\mathrm{S} / \mathrm{N}$ by much fainter comparison stars that need to be used from the ground.

In the following sections we briefly introduce the instruments on SOFIA that can be used for exoplanet spectrophotometry.

\subsubsection{HIPO}

The High Speed Imaging Photometer for Occultations (HIPO) is a Special Purpose Principal Investigator class Science Instrument (SSI, Dunham et al. 2004, 2014). HIPO is designed to provide simultaneous high-speed time resolved imaging photometry at two optical wavelengths. The HIPO field of view (FoV) is a $5.6^{\prime}$ square, the $8^{\prime}$ diagonal of which corresponds to the $8^{\prime}$ diameter SOFIA field of view. The filter set includes the Johnson $(U B V R I)$ and Sloan $\left(u^{\prime} g^{\prime} r^{\prime} i^{\prime} z^{\prime}\right)$ filters as well as a filter for methane at $890 \mathrm{~nm}$.

\subsubsection{FLITECAM}

The First Light Infrared Test Camera (FLITECAM) is a nearinfrared imager and grism spectrograph covering the $\sim 1-5 \mu \mathrm{m}$ range (McLean et al. 2006; Logsdon et al. 2014). The full set of available FLITECAM filter pass bands are listed online in the FLITECAM chapter of the SOFIA Observer's Handbook. FLITECAM was co-mounted with the HIPO instrument during these observations, a configuration that precluded observations at wavelengths longer than $\sim 4 \mu \mathrm{m}$, and reduced the sensitivity at wavelengths longer than $\sim 2 \mu \mathrm{m}$, due to high background levels resulting from the warm dichroic and transfer optics.

\subsection{3. $\mathrm{FPI}_{+}$}

On SOFIA the light passes through the telescope's dichroic tertiary mirror $\left(25 \%\right.$ and $45 \%$ reflectivity for the $B$ and $z^{\prime}$ bandpasses) to the Focal Plane Imager (FPI+, Pfüller et al. 2016). The FPI+ contains a highly sensitive and fast electron multiplying charge-coupled device (EM-CCD) camera. Its images are primarily used for tracking but can also be stored without disrupting the tracking process and in parallel with measurements of the instruments mounted to the telescope. With the released call for proposals for the SOFIA observing cycle 4 (2015), the FPI+ was made available for proposals as a facility science instrument for observations in 2016 and thereafter. 
Table 1. GJ1214 and reference stars in SOFIAs FPI+ field of view.

\begin{tabular}{llrc}
\hline \hline Star & 2MASS & $\begin{array}{c}K \\
(\mathrm{mag})\end{array}$ & $\begin{array}{c}\text { Dist } \\
(\operatorname{arcmin})\end{array}$ \\
\hline GJ 1214 & J17151894+0457496 & 8.782 & 0 \\
Ref. star 1 & J17152424+0455041 & 8.831 & 3.05 \\
Ref. star 2 & J17151760+0455021 & 10.318 & 2.81 \\
\hline
\end{tabular}

\section{Observation}

The joint US-German Cycle 2 Guest Investigator (GI) programme - US-proposal: Angerhausen (2013); German-proposal: Dreyer (2013) - was performed on SOFIAs flight number 149 on UT February 27, 2014. We observed the transit of the exoplanet GJ 1214b using the photometry mode of FLITECAM and HIPO in the "FLIPO" configuration in order to perform differential aperture photometry of the target and a bright comparison star (see Table 1 and Fig. 1). GJ 1214b was monitored during one 52 min transit plus ca. 70 min before and $10 \mathrm{~min}$ after transit for a total of $150 \mathrm{~min}$ (including some additional time for setups and calibrations). Observations were simultaneously conducted in two optical HIPO channels: open blue at $0.3-0.6 \mu \mathrm{m}$ and Sloan $z^{\prime}$ at $0.9 \mu \mathrm{m}$, and one infrared FLITECAM filter: Paschen- $\alpha$ cont. at $1.9 \mu \mathrm{m}$. The individual exposure time for all HIPO and FLITECAM frames was $25 \mathrm{~s}$. Complementary data were also obtained with the optical focal plane guiding camera FPI+ in the Sloan $i^{\prime}$ band $(0.8 \mu \mathrm{m})$ with mostly $2.5 \mathrm{~s}$ (but some with $3 \mathrm{~s}$ ) exposure time, as it was used for both tracking and data acquisition purposes. The change from $3 \mathrm{~s}$ to $2.5 \mathrm{~s}$ integrations was triggered by an increasing photon count at higher elevations. We chose to take shorter exposures to avoid saturation. We used an open filter for the HIPO blue side in order to cope with the faintness of GJ 1214 at blue wavelengths. This bandpass is defined by the transmission of the atmosphere, telescope, HIPO blue side optics, and the reflection curve of the internal HIPO dichroic reflector with a transition wavelength of $675 \mathrm{~nm}$. The HIPO red filter was selected to avoid possible telluric ozone variability, while the FLITECAM filter was chosen due to its wavelength coverage of a prominent $\mathrm{H}_{2} \mathrm{O}$ spectral feature that cannot be sampled from ground-based observatories. The filter selected for the FPI+ was an intermediate wavelength between the HIPO channels, which is somewhat ozone sensitive. This provided the potential for detecting and removing residual telluric ozone-related systematics from the HIPO $z^{\prime}$ filter. For this observation the FPI+ acquired images with a Sloan $i^{\prime}$ filter to complement the HIPO blue channel and Sloan $z^{\prime}$ filter. The Sloan $i^{\prime}$ filter has a central wavelength of $760 \mathrm{~nm}$ with a pass-band between $694 \mathrm{~nm}$ and $843 \mathrm{~nm}$. The average throughput with this filter is $24.4 \%$ taking into account a simulated atmosphere at flight altitude, the reflectivity and transmittance of all optical elements in the light path through the telescope, and the CCD sensor quantum efficiency. In addition to the acquisition of science data, the FPI+ was simultaneously used as a tracking camera to keep the telescope precisely pointed at the target. The tracking accuracy, as measured with the FPI+, was 0.17 arcsec rms. Full frame images $(1024 \times 1024$ pixel $)$ were taken with a $2 \times 2$ pixel binning, which resulted in a spatial resolution of 1.03 arcsec per pixel and a square field of view of 8.8 arcmin. The image integration time was set to $2.5 \mathrm{~s}$ to achieve maximum pixel values at about $65 \%$ full well capacity of the sensor. The HIPO instrument was operated in Basic Occultation mode with full-frame read-out to maximise our field standard possibilities. We used simple stare mode to minimise

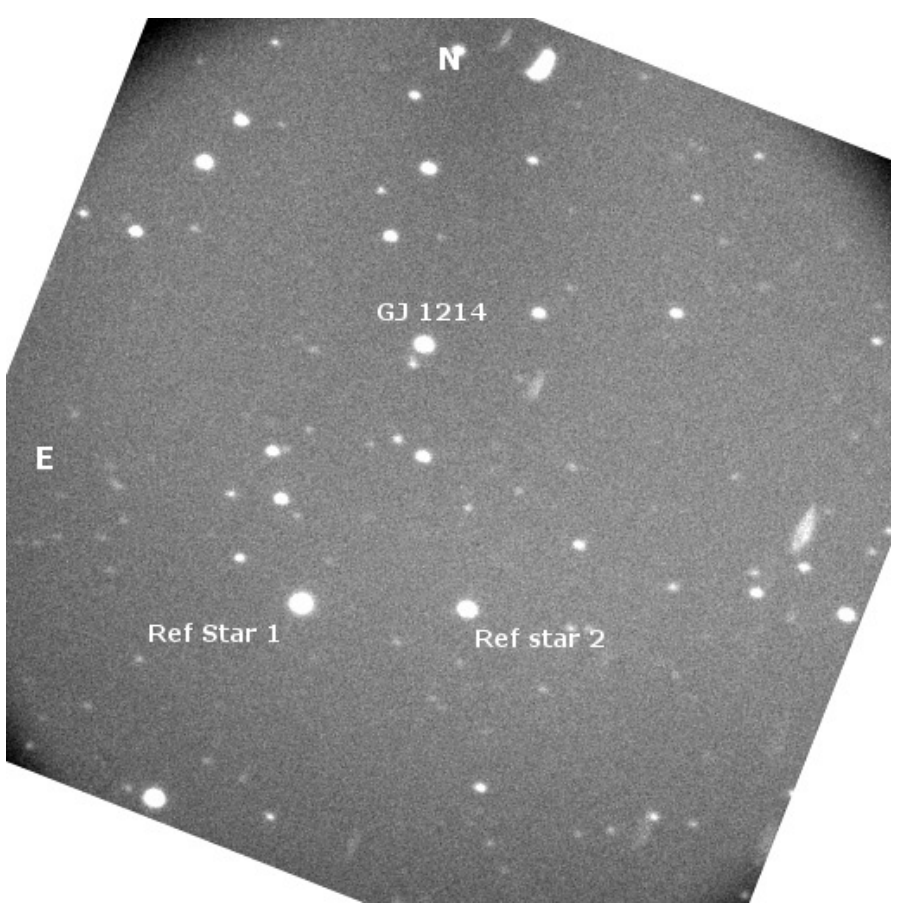

Fig. 1. SOFIAs $8^{\prime} \times 8^{\prime}$ FPI+ field of view showing our target GJ 1214 and reference stars.

the contribution of systematic errors. In addition, slight defocussing was applied in order to minimise the potential instability problems with telescope guiding and to increase the $S / N$ ratio. Bias and all other frames were taken at $512 \times 512$. Table 2 gives an overview of the final data set.

Due to flight planning constraints, the end of the transit occurred in morning twilight and in the last $\sim 15 \mathrm{~min}$ the sky brightness gradually increased to about 3.5 times its night-time values. In Sect. 3.3.2 we describe how we correct for the changing observational parameters in general via a principal component analysis method; in Sect. 3.3.3 we describe how we had to correct for the twilight contribution to the HIPO blue channel.

\section{Data reduction and analysis}

\subsection{Light curve extraction}

Standard data reduction was applied to the data taken with HIPO (red and blue) and FPI+. This includes bias and dark subtraction, and in the case of FPI+ also flat field correction. For FLITECAM we did not acquire bias frames, as bias contributions are generally very low for this type of NIR detector array. Furthermore, it is complicated and time consuming to obtain a reliable flat field on such a narrow band filter as the $1.9 \mu \mathrm{m}$ Paschen- $\alpha$ continuum filter. Since it was not possible to take long enough exposures during this campaign, we used $K$-band flat fields taken on the same flight before our observation run, which, however, did not improve the photometric precision significantly. Similarly, dark subtraction did not show any improvement in photometric precision. Additionally, we corrected for the sky background using dithered images taken during the observation run. Stars were detected with the Source Extractor by Bertin \& Arnouts (1996). Aperture photometry was applied using Image Reduction and Analysis Facility's (IRAF Tody 1993) DAOPHOT (Stetson 1987) using circular apertures. The optimal aperture radius with the lowest noise level was found to be six pixels for FLITECAM and 12 pixel for HIPO and FPI+. As part 
Table 2. Observation summary.

\begin{tabular}{lcccc}
\hline \hline & \multicolumn{2}{c}{ HIPO } & \multirow{2}{*}{ FPI+ } & \multirow{2}{*}{ FLITECAM } \\
\cline { 2 - 3 } & HIPO-blue & HIPO-red & \\
\hline Observation time [UTC] & \multicolumn{3}{c}{$2014-02-27,10: 00: 55-13: 43: 33$} \\
Pass-band* $^{*}$ open blue & SDSS $z^{\prime}$ & SDSS $i^{\prime}$ & Pa $\alpha$ cont. \\
$\lambda_{\text {eff }}[\mu \mathrm{m}]^{*}$ & $0.3-0.7$ & 0.89 & 0.76 & 1.90 \\
${\text { Band-width }[\mu \mathrm{m}]^{*}}_{\text {Exposure time [s] }}$ & 0.4 & 0.23 & 0.15 & 0.02 \\
No. of frames & 25 & 25 & $2.5 / 3$ & 25 \\
Image [px] & 459 & 448 & $4786(2882 / 1904)$ & 433 \\
Dark [px] & $512 \times 512$ & $512 \times 512$ & $1024 \times 1024$ \\
Flat [px] & $512 \times 512$ & $512 \times 512$ & - \\
Bias [px] & $512 \times 512$ & $512 \times 512$ & - \\
\hline
\end{tabular}

Notes. ${ }^{*}$ Taken from Sofia Observer's Handbook for Cycle 2: v2.1.2.

of the DAOPHOT routine, an annulus around the target was used to estimate the sky background in each exposure. Next to GJ 1214 , we extracted the light curves of two additional bright stars within our field of view (see Table 1 and Fig. 1). To identify stars in the images we calculated a rough astrometric solution for each image using data provided by Astrometry.net (Barron et al. 2008). In the FLIPO setup SOFIA does not provide an image rotator to compensate field rotation during long integrations. This introduces a rotation of the images over time. Due to SOFIA's unique setup, the telescope must periodically undergo so-called "line-of-sight (LOS) rewinds". The required frequency of LOS rewinds depends on the rate of field rotation experienced by the target, which is a complex function of the position of the target in the sky relative to that of the aircraft heading. These need to be carefully timed with regard to the transit observation, to not interfere with, for example, ingress or egress. While we kept the target star, GJ 1214, in boresight, the comparison stars moved over the CCD due to this field rotation. This is one of the main factors introducing systematic noise and limiting the photometric precision of the instrument and is another reason why reliable flat fields are crucial for this kind of time series observation. After comparing the light curves, the brighter star was selected as comparison star to correct for first-order systematic effects present in all light curves.

\subsection{Observational parameters in the airborne environment}

Photometric observations from an airborne platform like SOFIA differ from ground-based observations. While ground-based photometry suffers from systematic errors induced by e.g. air mass or local weather changes, photometric observations with SOFIA also correlate with changes in flight parameters such as Mach-number or air density.

We used the housekeeping data taken during our observation to parameterize the time dependence of our observational environment. Figure 2 shows time series of some selected observational parameters, some of them unique to the airborne environment. Many of these parameters are mutually correlated. In order to overcome these degeneracies we performed a principal component analysis on all available parameters to produce a set of linearly independent time series to eventually decorrelate the raw light curves (see Sect. 3.3.2).

\subsection{Light-curve and noise modeling}

In this section we present two methods that were used to fit the resulting light curves. In the second case we also present a method to correct for the aforementioned systematics induced by the airborne environment.

\subsubsection{TLCM applied to the raw light curves}

The main features of the Transit Light Curve Modeller (TLCM) code are described in Csizmadia et al. (2011, 2015). Therefore we repeat only the most important pieces of information about TLCM here. The TLCM uses the formalism of Mandel \& Agol (2002), which is based on spherical star and planet shapes, to fit the light curves. To optimise the fit, first a genetic algorithmbased Harmony Search (Geem et al. 2001) was performed, then an Amoeba algorithm refined the fit (Press et al. 1992). Finally we used simulated annealing (SA, Press et al. 1992, and references therein) for error estimation as well as to better monitor the possible parameter correlations. The SA chain consisted of $10^{5}$ steps. The SA process is quite similar to the Markov Chain Monte Carlo (MCMC). However, in SA the control parameter (the so-called "temperature" of the Metropolis-Hastings procedure of MCMC) is continuously and slowly decreased. When this "temperature" is very small, then SA will be similar to a simple random walk, and when it is large then it is equivalent to MCMC. We decreased this temperature by $1 \%$ after every 2000 steps starting from such a value that in this way we reached the point at which the overall acceptance rate was around $30 \%$.

The host star, GJ 1214, is a chromospherically active Mdwarf (Nascimbeni et al. 2015). Thus spot activity may affect the light-curve fit in several different ways. The following effects seem to be important:

a) The rotational modulation of the stars caused by spots and stellar rotation yields a long-term oscillation of the light curve that has a much longer timescale (days or weeks) than the length of the transit or our observational window. This effect was removed with a parabolic baseline fit.

b) Spot-crossing during the transit (see e.g., 2011; SanchisOjeda \& Winn Silva-Valio \& Lanza 2011) severely affected other observations of GJ 1214b's transit (e.g. Bean et al. 2011). However, there is no clear sign of spot-crossing at our epoch of observation or it is lost in the noise. 

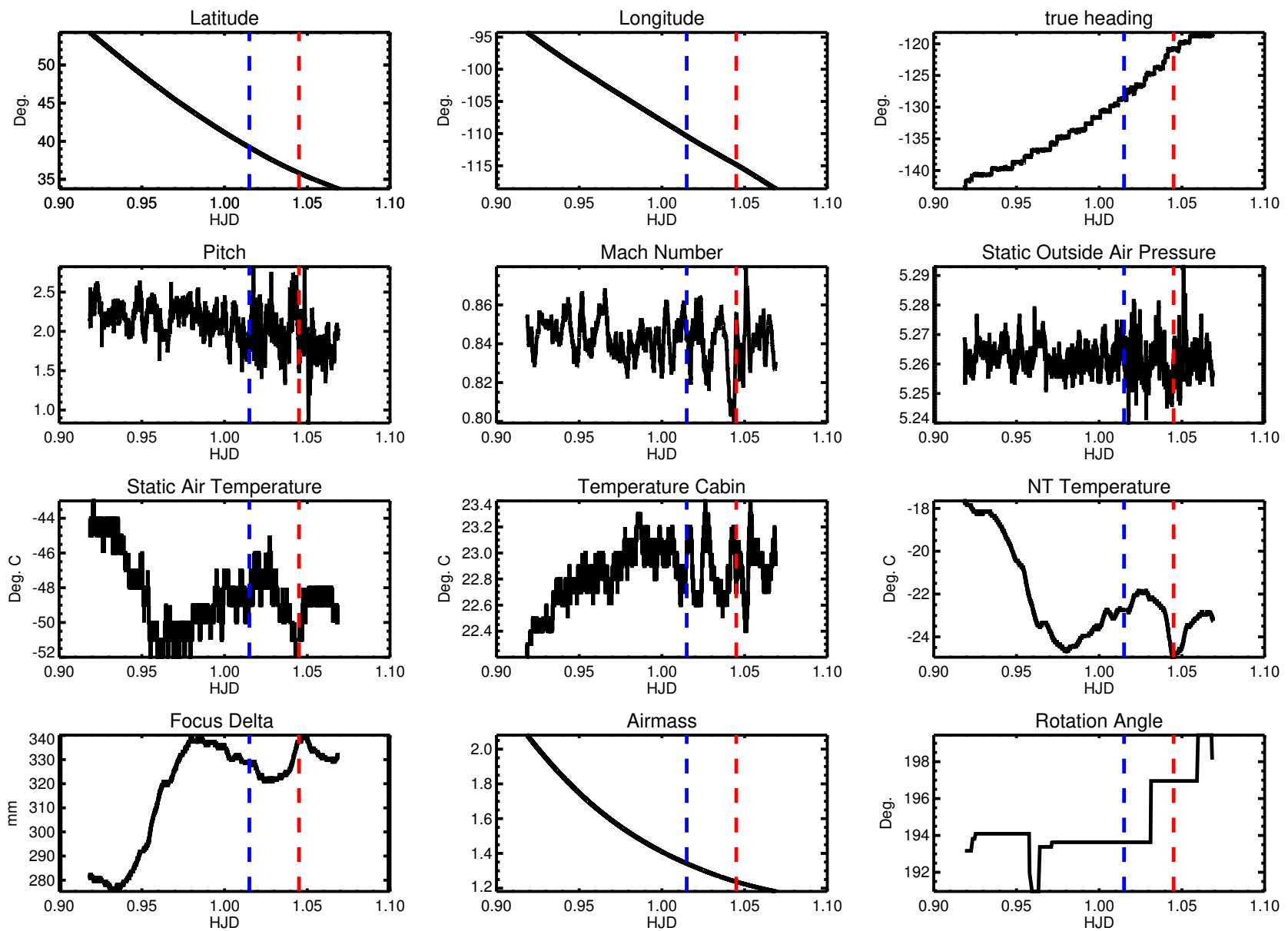

Fig. 2. Sample time series of some observational parameters. While parameters like the plane heading or pitch should not influence the photometry, they illustrate the mobile airborne environment. The beginning of ingress and end of egress are marked in blue and red vertical lines.

c) Spots that do not cross and/or are polar spots will cause changes in the observable limb darkening coefficients (Csizmadia et al. 2013b). In addition, the theoretically predicted limb darkening coefficients, especially the recent tables, have not been observationally verified. That is why, following the recommendation of Csizmadia et al. (2013b) and Espinoza \& Jordán (2015), we adjusted the limb darkening coefficients.

The long-term behaviour of the stellar variability and the estimated effect of the stellar spots on the systematic and random errors in the derived planet-to-stellar radius ratios, which were not removed by the baseline-fit, are discussed separately in Sect. 3.4.

The TLCM-based light-curve modelling was carried out by fixing the scaled semi-major axis at $a / R_{\mathrm{s}}=14.97$ and the impact parameter at $b=0.277026$. These values were chosen to match the values used by other investigators (e.g. Cáceres et al. 2014; Désert et al. 2011; Croll et al. 2011; Bean et al. 2011; de Mooij et al. 2012; Murgas et al. 2012; Narita et al. 2013b,a; Fraine et al. 2013; Teske et al. 2013) so that our results are more readily comparable to those works.

We also note that the eccentricity of GJ $1214 \mathrm{~b}$ is not well constrained (e.g. Charbonneau et al. 2009, gives only an upper limit for eccentricity of $e<0.27$ ). The impact of eccentricity on the light-curve fit is not investigated by other authors, who all assume a circular orbit. However, eccentricity has an effect on the speed of the planet during transit and thus on $a / R_{\mathrm{S}}$. Therefore, the stellar density measured from the transit duration is somewhat approximate. Consequently, the stellar parameters should also be considered approximate until the eccentricity has been established. Since we have only one photometric transit measurement and no additional radial velocity follow-up data, we are also not in the position to further constrain the eccentricity. Therefore, we decided to use a circular orbit for the fit as other authors do because the analysis can be repeated later if a significant eccentricity is found. We urge the community to collect more radial velocity data points to finally close the eccentricity issue of GJ $1214 \mathrm{~b}$.

The Mandel \& Agol (2002) formalism calculates the planetstar mutual distance projected to the sky as

$\delta=a / R_{\mathrm{s}}(\cos \Omega t+\sin \Omega t \sin i), \quad \Omega=2 \pi \frac{t-E}{P}$,

where $P$ is the orbital period, $E$ is the epoch, $t$ is the time, and $i$ is the inclination. The equation above is valid for circular orbits. This can be easily generalised to eccentric orbits (e.g., Russell 1912; Giménez 2006):

$\delta=a / R_{\mathrm{s}} \frac{1-e^{2}}{1+e \cos v} \sqrt{1-\sin ^{2} i \sin (v+\omega)}$,

where $e$ is the eccentricity, $\omega$ is the argument of periastron, and $v$ is the true anomaly calculated from the solution of the Keplerequation.

However, as we mentioned, the eccentricity is not well known for GJ $1214 \mathrm{~b}$ and this may significantly affect the end result. Therefore we used the following equation instead of the one 
Table 3. Results of the light-curve modelling.

\begin{tabular}{|c|c|c|c|c|c|}
\hline & \multicolumn{3}{|c|}{ HIPO } & \multirow[t]{2}{*}{ FPI+ } & \multirow[t]{2}{*}{ FLITECAM } \\
\hline & HIPO-blue & HIPO-blue, short & HIPO-red & & \\
\hline $\begin{array}{l}\text { Photon noise limited } \\
\text { sensitivity }[\mathrm{ppm} / \mathrm{min}]\end{array}$ & \multicolumn{2}{|c|}{1000} & 400 & 500 & 1000 \\
\hline ppm per exposure & \multicolumn{2}{|c|}{$1600(25 s)$} & $650(25 s)$ & $2500 / 2220(2.5 / 3 \mathrm{~s})$ & $1600(25 \mathrm{~s})$ \\
\hline $\begin{array}{l}P \text { [days }] \\
a / R_{\mathrm{s}} \\
i \text { degree }] \\
e \\
b\end{array}$ & \multicolumn{5}{|c|}{$\begin{array}{l}1.5804055929 \text { (fixed) } \\
14.9749 \text { (fixed) } \\
88.94 \text { (fixed) } \\
0 \text { (fixed) } \\
0.27702737 \text { (fixed) } \\
\end{array}$} \\
\hline \multicolumn{6}{|c|}{ TLCM fitting with polynomial correction } \\
\hline $\begin{array}{l}k=R_{\mathrm{p}} / R_{\mathrm{S}} \\
u+ \\
u- \\
\text { epoch }\end{array}$ & $\begin{array}{ll}0.1281 & \pm 0.003 \\
0.541 & \pm 0.293 \\
0.497 & \pm 0.81 \\
1.03419 & \pm 0.0005\end{array}$ & $0.1184 \pm 0.0189$ & $\begin{aligned} 0.1156 & \pm 0.0023 \\
0.572 & \pm 0.19 \\
-0.189 & \pm 0.35 \\
1.03304 & \pm 0.0003\end{aligned}$ & $\begin{array}{ll}0.1133 & \pm 0.0029 \\
0.747 & \pm 0.293 \\
0.143 & \pm 0.73 \\
1.03313 & \pm 0.0003\end{array}$ & $\begin{aligned} 0.1203 & \pm 0.0046 \\
0.35 & \pm 0.30 \\
-0.71 & \pm 0.83 \\
1.0328 & \pm 0.0005\end{aligned}$ \\
\hline$\chi^{2}$ of the fit & & & 1.2040 & & \\
\hline \multicolumn{6}{|c|}{ EXONEST fitting with principal component noise correction* } \\
\hline $\begin{array}{l}k=R_{\mathrm{p}} / R_{\mathrm{s}} \\
u_{1} \\
u_{2}\end{array}$ & $\begin{array}{ll}0.1288 & \pm 0.0028 \\
0.704 & \pm 0.292 \\
0.011 & \pm 0.280 \\
\end{array}$ & $0.1225 \pm 0.0017$ & $\begin{array}{cl}0.1156 & \pm 0.0026 \\
0.677 & \pm 0.294 \\
-0.170 & \pm 0.280 \\
\end{array}$ & $\begin{array}{cl}0.1107 & \pm 0.0011 \\
0.864 & \pm 0.228 \\
-0.481 & \pm 0.220 \\
\end{array}$ & $\begin{array}{l}0.1215 \pm 0.005 \\
0.253 \pm 0.186 \\
0.365 \pm 0.254 \\
\end{array}$ \\
\hline$\chi^{2}$ of the fit & \multicolumn{5}{|c|}{1.3245} \\
\hline Final $k=R_{\mathrm{p}} / R_{\mathrm{s}}$ & \multicolumn{2}{|c|}{0.1246} & 0.1156 & 0.1107 & 0.1215 \\
\hline $\begin{array}{l}1 \sigma \text { (formal) } \\
2 \sigma \text { (incl. syst.) } \\
1 \sigma \text { (theo. noise limit) }\end{array}$ & \multicolumn{2}{|c|}{$\begin{array}{l} \pm 0.0037 \\
\pm 0.0074 \\
\pm 0.0017\end{array}$} & $\begin{array}{l} \pm 0.0026 \\
\pm 0.0052 \\
\pm 0.0012\end{array}$ & $\begin{array}{l} \pm 0.0011 \\
\pm 0.0022 \\
\pm 0.0007\end{array}$ & $\begin{array}{c} \pm 0.005 \\
\pm 0.01 \\
\pm 0.002\end{array}$ \\
\hline
\end{tabular}

Notes. The epochs are relative to JD 2456715.0. * EXONEST did not fit the epoch explicitly but instead the mean anomaly at epoch.

recommended by Mandel \& Agol (2002) to describe the skyprojected star-planet distance (Csizmadia et al. 2013a):

$\delta=a / R_{\mathrm{s}} \times \sqrt{b^{2}+(t-E) \cdot\left((1+k)^{2}-b^{2}\right) / P}$.

The Csizmadia et al. (2013a) equation is based on the assumption that the planet moves with constant projected velocity during transit. We then interpolate the planet's motion linearly. This assumption is quite good even for high eccentricities and closein orbits where the transit light curve would become asymmetric due to the slowly changing projected velocity. However, at the present level of photometric accuracy the asymmetry should not be taken into account (cf. Moutou et al. 2009, who did not find this asymmetry in the transit of HD $80606 \mathrm{~b}$ whose eccentricity is 0.93 ).

Our free parameters were: four planet-to-stellar radius ratios (one for each of the four pass-bands we observed in), the four corresponding $\mathrm{u}+$ and $\mathrm{u}-$ limb darkening coefficient combinations, and the four epochs of observations (again, one for each pass-band). The limb darkening combinations were defined as $u+=u_{1}+u_{2}$ and $u-=u_{1}-u_{2}$, where $u_{1}$ is the linear and $u_{2}$ is the quadratic term of the quadratic limb darkening law. According to Brown et al. (2001) and Pál (2008), such combinations are less sensitive to degeneracies between the coefficients. We decided to leave these parameter combinations as free parameters, because no theoretical limb darkening calculations are available for the instruments and pass-bands we used. In addition, the host star is a convective, active M-dwarf and theoretical calculations do not include the stellar spots so far, nor the probable exciting granulation pattern of small stars. We divided the data by a parabola whose coefficients were fitted simultaneously with the light-curve parameters. This parabola served as our baseline-corrections to remove any stellar activity signal or long timescale instrumental and air mass effects.

A simultaneous fit to the different data sets would have the advantage that the number of free parameters is decreased, because the wavelength-independent parameters would be the same for every data set. However, the epoch of observation is not necessarily the same in all colours because of the distribution of data points or because the planetary atmosphere is asymmetric. A planet with a non-spherical atmosphere may have different atmospheric density and thus atmospheric transparency causing a slightly asymmetric transit shape (e.g. if it loses its atmosphere). If the number of data points is not symmetric to the mid-point of the transit, for example if there is an unfortunately placed gap, then the timing error increases and the fitted mid-point can be shifted (e.g. Csizmadia et al. 2010). Therefore we fitted the four epochs but we found they are in good agreement with each other (Table 2). In total, we had 28 free parameters for the four pass-bands: four epochs, four planet-to-stellar radius ratios, twotimes-four limb darkening coefficients combinations, and fourtimes-three coefficients of the parabola.

Using the following ephemeris ${ }^{1}$ :

Transit $_{\mathrm{N}}=$ HJD $2454980.748795+1.58040482 \times N$

and transforming the observed transit times of Table 3 into HJDs, we found that we observed transit $N=1098$ and the

1 http://var2.astro.cz/ETD 


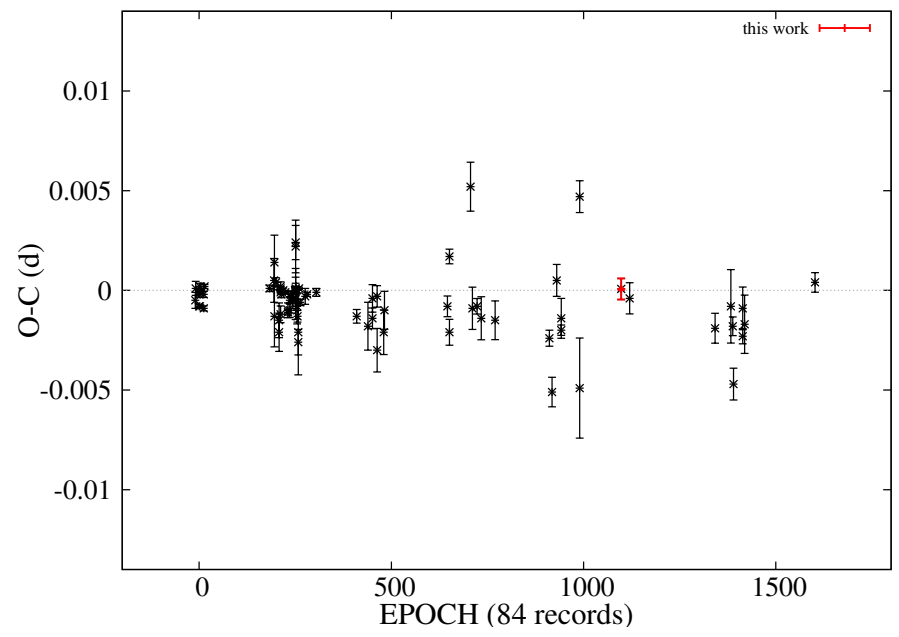

Fig. 3. O-C diagram of GJ 1214b. The red point marks our measurement. Black points are taken from Poddaný et al. (2010) using all (professional and citizen science) data from the Exoplanet Transit Database. For example the point at $\mathrm{O}-\mathrm{C}=0.005$ comes from one of the TRESCA light curves. Our data is consistent with other measurements finding no significant long-term variations in transit timing.

corresponding $\mathrm{O}-\mathrm{C}$ value is $+0.00007 \pm 0.00053$ days (cf. Fig. 3). The results of the fit are shown in tabular form in Table 3 and are visualised in Fig. 5 and are in perfect agreement with the ephemeris.

\subsubsection{EXONEST combined with principal component analysis}

We also tested an alternative fitting and decorrelation methodology analogous to the one used in the first SOFIA exoplanet observation by Angerhausen et al. (2015), where they used the Transit Analysis Package (TAP; Gazak et al. 2012) built on EXOFAST (Eastman et al. 2013) in two steps combined with an intermediate decorrelation of the (airborne) observational parameters (see e.g. Fig. 2). Here we use the same approach, but replace TAP by a Bayesian nested sampling fit with EXONEST.

EXONEST is a Bayesian inference tool aimed at characterising exoplanets through Bayesian model selection and parameter estimation (Placek 2014; Placek et al. 2014, 2015; Placek \& Knuth 2015). This tool allows one to analyse an assortment of exoplanetary data using a variety of inference engines such as nested sampling (Sivia \& Skilling 2006), multinested sampling (Feroz et al. 2009, 2011, 2013), MetropolisHastings Markov chain Monte Carlo (MCMC) sampling (Metropolis et al. 1953), and simulated annealing (Otten \& van Ginneken 1989). Multi-nesting was chosen in this specific analysis for its efficiency in sampling from complicated parameter spaces. Inputs to EXONEST consist of the prior probabilities for each model parameter, which reflect one's knowledge of the model parameters prior to having analysed the data, and the likelihood function, which depends on the model and the expected nature of the noise.

The four channels of photometric time series obtained from SOFIA were simultaneously fitted using the model of Mandel \& Agol (2002), which is parametrised by the planetto-star radius ratio, and quadratic limb darkening coefficients for each channel, the scaled semi-major axis, $a / R_{\star}$, and the impact parameter $b=\frac{a}{R_{\star}} \cos i$. The planet-to-star radius ratio, quadratic limb darkening coefficients, and the impact parameter were each sampled from uniform prior probability distributions over the ranges $[0,0.2],[0,1]$, and $[0,1]$, respectively. For more straightforward comparisons to other methods, we again fixed the scaled semi-major axis at $a / R_{\mathrm{S}}=14.97$, the impact parameter at $b=0.277026$, the orbital period to $P=1.5804055929$ days, and the eccentricity to zero. Assuming the noise in each channel to be Gaussian distributed, the likelihood function for each channel, $L$, takes on the form:

$$
L=\sum_{i=1}^{N} \frac{1}{\sqrt{2 \sigma_{i}^{2}}} \exp \left(-\frac{\left(F_{i}-d_{i}\right)^{2}}{2 \sigma_{i}^{2}}\right),
$$

where $N$ is the number of data points in the channel, $\sigma_{i}$ is the standard deviation of the $i$ th data point $d_{i}$, and $F_{i}$ is the corresponding model prediction. MultiNest works to maximise the likelihood (or log-likelihood) function to ultimately obtain the posterior distribution from which parameter estimates can be derived.

Prior to fitting, a 3- $\sigma$ clipping was performed on the raw data for the removal of outliers. Following the method in Angerhausen et al. (2015), the transits were then modelled in three steps. First, the raw light curves were fitted with a transit model and a second-order polynomial to account for airmass. The residuals to the initial fits were then modelled with the first 16 principal components $p_{i}(t)$ in order to decorrelate with the observational parameters, sampled at the same time as our exposures, as a linear combination $R_{\text {model }}(t)=\sum c_{i} \times p_{i}(t)$. Figure 4 displays the raw data with the transit+quadratic fits, and the corresponding residuals (again including the quadratic) with these principal component fits. This noise model was computed independently from the iterative EXONEST analysis and no marginalisation has been done over the instrument model correlation terms. As also argued in Angerhausen et al. (2015), we chose this approach because the lack of post transit baseline causes convergence issues and a high risk of running into degeneracies between the noise model and the actual transit depth.

Finally, the best-fit principal component model for each channel was subtracted from the raw data, and the (decorrelated) transits were fitted again. The results of these simulations are displayed in Table 3, and the corresponding principal component analysis (PCA) noise models are shown in grey in Fig. 4. The estimated planet-to-star radius ratios are in good agreement with the results from TLCM in Sect. 3.3.1.

\subsubsection{Correction of twilight effects on HIPO-blue light curve}

In both reductions, the methods used to account for systematic noise (polynomial slope, linear combination of principal components) failed to account for an upward trend in the HIPO-blue post-transit observations. These observations were taken close to sunrise and affected the shortest wavelength channel significantly towards the end of the flight. This led to a transit depth several standard deviations from previous measurements. To investigate whether this post-transit slope was skewing the estimated transit depth, a series of simulations were performed with an increasing amount of post-transit data neglected. The results of this process are displayed in the left-hand panel of Fig. 7. For the PCA corrected data, after thirty points were neglected from the post-transit observations, the derived value for the transit depth began to plateau. The resulting value associated with thirty post-transit points neglected is $R_{\mathrm{p}} / R_{\mathrm{S}}=0.122537$ for the PCA corrected data. For the TLCM fits to the raw data, the results did not plateau but instead consistently decreased as more data 

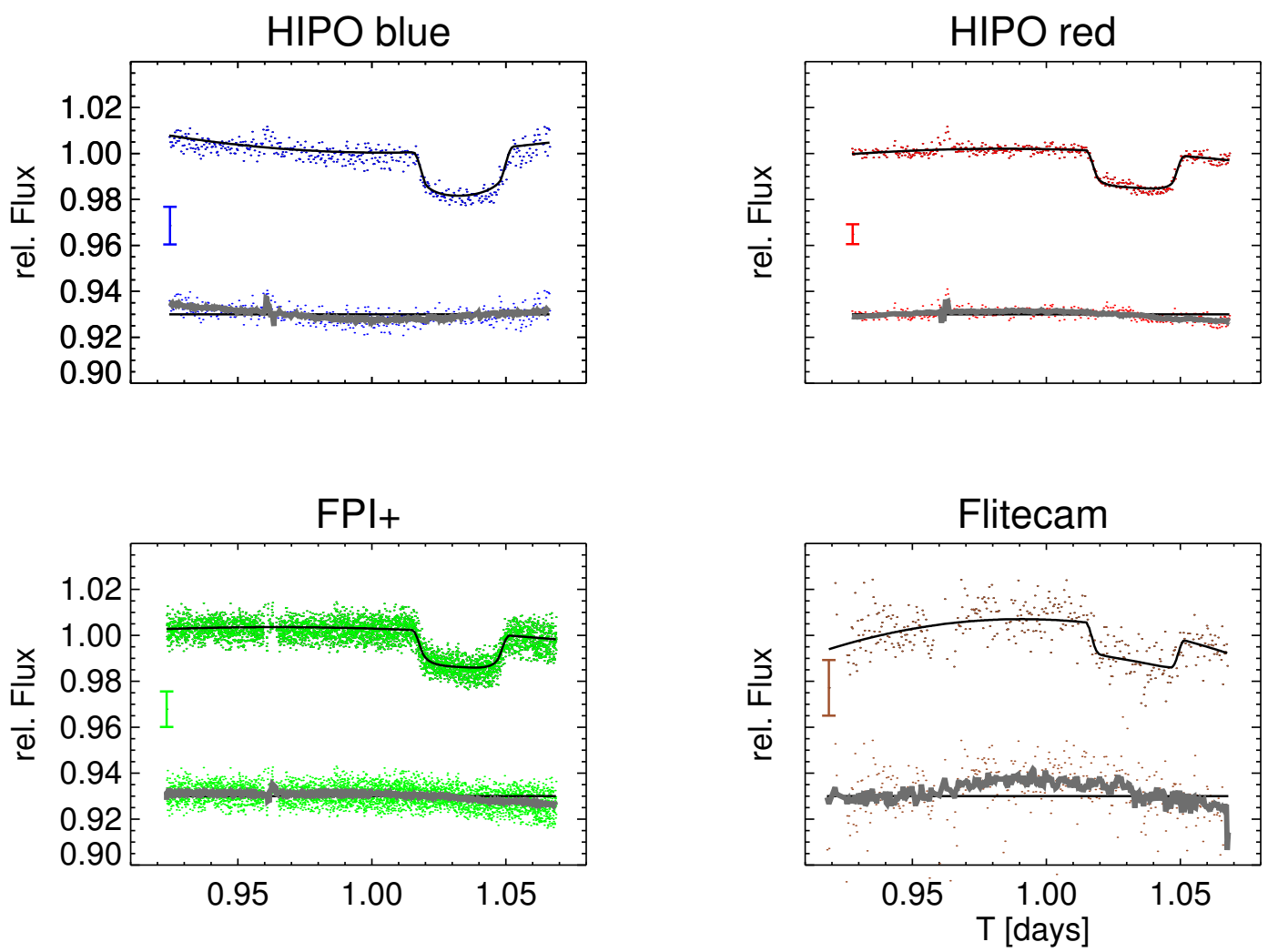

Fig. 4. Initial fits (transit model + quadratic) to the raw data (outliers removed) in black. The later applied principal component noise model (grey) is overplotted to the residuals. Top left, blue: HIPO blue; top right, red: HIPO red; bottom left, green: FPI+; bottom right, brown: FLITECAM.

points were neglected to a final value as low as $R_{\mathrm{p}} / R_{\mathrm{s}}=0.11845$. In a conservative approach we therefore decided to report the whole range of possible outcomes as our final values for HIPO blue in both methods (see left panel of Fig. 7 and Table 3) and with the $2 \sigma$ error to account for the systematic contribution in addition to the formal $1 \sigma$ error.

\subsection{Influence of host star activity}

Star spots not crossed by the transiting planet cause the average brightness to be higher along the transit chord than on the rest of the stellar hemisphere, which leads to an overestimation of the planet-star radius ratio (Csizmadia et al. 2013b; Czesla et al. 2009). This effect is wavelength dependent due to the different temperatures and spectral energy distributions of spotted and unspotted photospheres (Pont et al. 2008; Sing et al. 2011). The host star GJ 1214 was photometrically monitored in the observing season 2014 with the robotic telescope STELLA and its Wide Field STELLA Imaging Photometer (WiFSIP; Strassmeier et al. 2004). The observations continued the WiFSIP monitoring programme of 2012 and 2013 presented in Nascimbeni et al. (2015). Details of the observations and data reduction are presented in Mallonn et al. (in prep.). The longterm photometry proved the host star to be at maximum brightness at the time of the SOFIA transit observation. If maximum brightness was interpreted as a spot-free visible hemisphere, no correction of the derived transit parameters would be needed. However, the monitoring only yields information on the relative change in spot filling factor, but no information about the level of spots permanently visible. Nascimbeni et al. (2015) estimated the differential correction for a filling factor of $2 \%$ between Bessel B and Bessel $\mathrm{R}$ to $<0.0001$ in the planet-star radius ratio $\mathrm{k}$. If we conservatively assume a permanent spot filling factor of $4 \%$ (which is on the order of the maximum change in the spot filling factor in the season 2014), it results in a differential spot correction of 0.0002 in the optical, which is an order of magnitude smaller than our error bars for $\mathrm{k}$. Since the value of the correction further decreases towards the near infrared, we conclude that a correction for un-occulted spots is negligible in our case. We note that the monitoring light curve of 2014 displays the largest amplitude and longest apparent periodicity ever observed for the super-Earth host star GJ 1214. We refer to Mallonn et al. (in prep.) for an in-depth analysis of five years of GJ 1214 monitoring from 2012 to 2016.

\subsection{Noise analysis}

In Fig. 6 we show the variance of the residuals as a function of bin size. This scheme is commonly used in the literature to assess the amount of correlated noise and as a visual test of the noise correction method. While the raw data in both plots show strong deviation from the theoretical limit (dashed lines, $\sigma^{2}(l)=$ $\left.\sigma^{2}(0) / l\right)$ for pure white noise, they show that the PCA corrected data is much closer to the expected line. The red lines in these figures mark the length of the transit and are the frequencies that eventually bias the results most. This improvement shows that a large amount of the time-correlated components in the time series of residuals were removed after the correction via PCA, and could explain the discrepancy with the TLCM fitted results. However, Cubillos et al. (2017) report a number of caveats for using variance plots as a measure for residual correlated noise, as well as for other frameworks dealing with systematic noise such as the residual-permutation or wavelet-likehood methods. 
HIPO blue

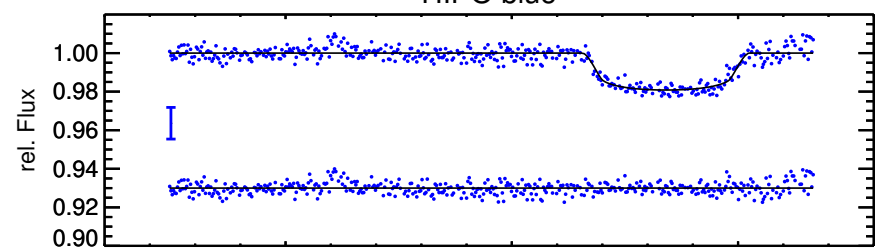

HIPO red

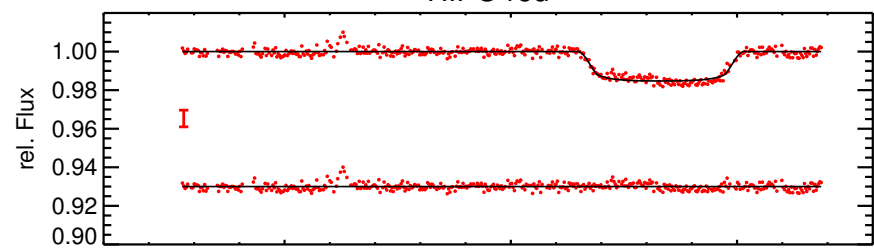

$\mathrm{FPI}+$

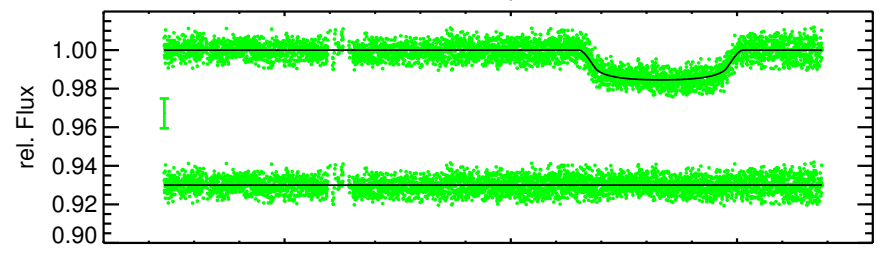

Flitecam

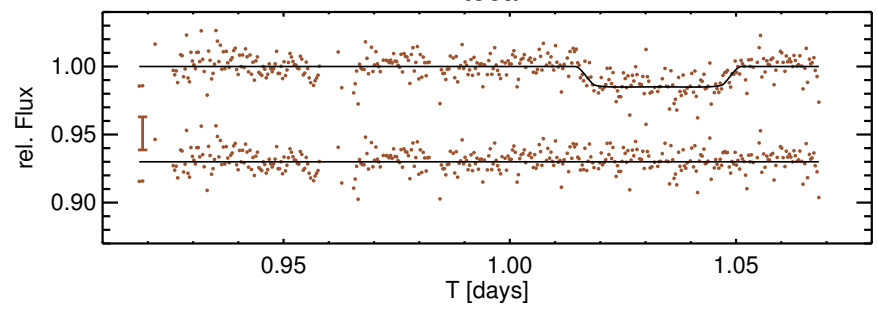

HIPO blue

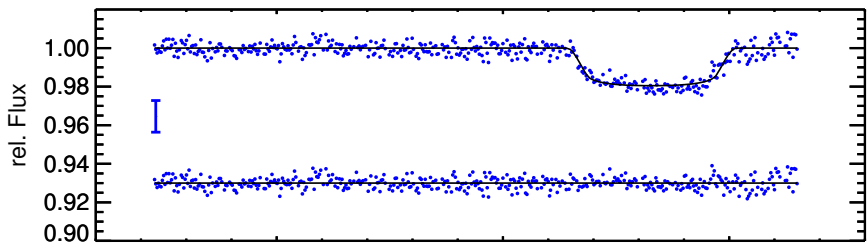

HIPO red

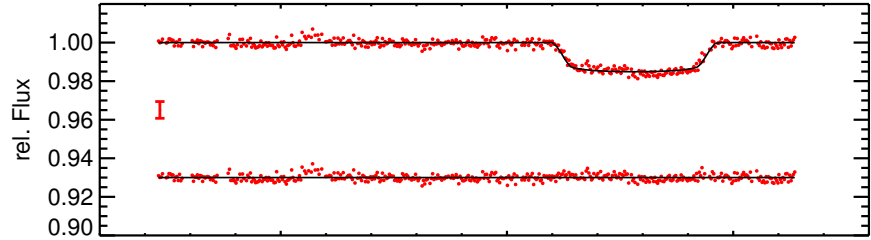

$\mathrm{FPI}+$

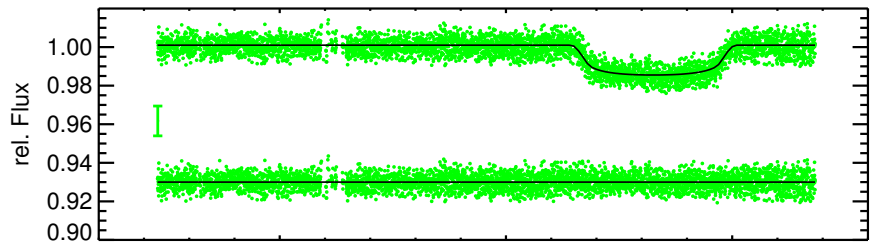

Flitecam

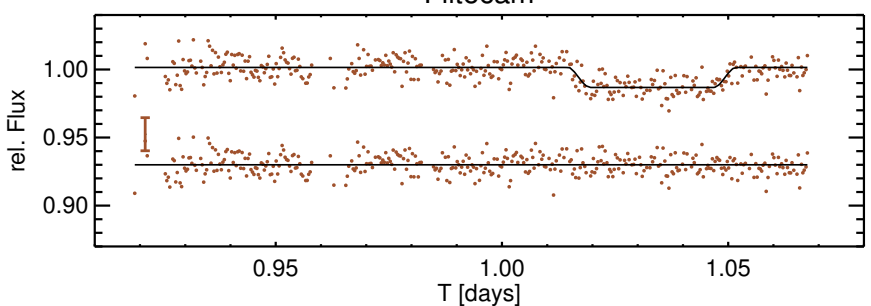

Fig. 5. Left: results of the TLCM fit to the raw data including a polynomial first-order noise model. Right: final EXONEST fits to the data reduced using a principal component analysis noise model.

In an alternative hands-on test to explore how close we approach the photon noise limit, we simulated a data set with white noise added according to the values derived from the raw data. As shown in Table 3, we obtain sensitivities of $500 \mathrm{ppm} / \mathrm{min}$ for FPI+, $400 \mathrm{ppm} / \mathrm{min}$ for HIPO-Red, $1000 \mathrm{ppm} / \mathrm{min}$ for HIPO blue, and $1000 \mathrm{ppm} / \mathrm{min}$ for FLITECAM using the gain values provided by the instrument teams. After running the same reduction and fitting procedure with the theoretical data, we obtain a factor of 2.2/2.2 for HIPO (blue/red), 1.6 for FPI, and 2.5 for FLITECAM smaller error bar for the derived transit depth compared to the formal $1-\sigma$ error of the real data fits for the transit depth. This is consistent with the findings in Angerhausen et al. (2015), where they reached approximately two times the photon noise. We decided to additionally report the $2 \sigma$ error with our final result to account for any residual systematic.

\section{Transmission spectra modelling}

For the calculation of the theoretical transmission spectra we follow the methods described in Gaidos et al. (2017). We adopt selected scenarios from Kreidberg et al. (2014), namely a waterrich case with $99 \% \mathrm{H}_{2} \mathrm{O}$ and $1 \% \mathrm{H}_{2} \& \mathrm{H}_{\mathrm{e}}$, as well as a hydrogen-dominated atmosphere composed of $99 \% \mathrm{H}_{2} \& \mathrm{H}_{\mathrm{e}}$ and $1 \%$ water. The water opacity is calculated with HELIOS-k (Grimm \& Heng 2015), employing the HITEMP2010 line list (Rothman et al. 2010). Collision induced absorption from HITRAN2010 is used for $\mathrm{H}_{2}-\mathrm{H}_{2}$ and $\mathrm{H}_{2}-\mathrm{H}_{\mathrm{e}}$ collisions. The molecular scattering cross sections are derived via the Rayleigh scattering equation

$\sigma_{\text {rayleigh }}=\frac{24 \pi^{3} v^{4}}{n_{\text {ref }}^{2}} \times\left(\frac{n(v)^{2}-1}{n(v)^{2}+2}\right)^{2} \times K(v)$,

where $v$ is the wavenumber, $n$ the refractive index, $n_{\text {ref }}$ a reference particle number density, and $K$ the King factor. The corresponding data for $\mathrm{H}_{2}$ is taken from Cox (2000), for $\mathrm{H}_{2} \mathrm{O}$ from Wagner \& Kretzschmar (2008) and Murphy (1977), and from Sneep \& Ubachs (2005) in the case of $\mathrm{H}_{\mathrm{e}}$. For the scenarios that include high-altitude hazes, we assume that the haze particles are composed of small hydrocarbon clusters (Kreidberg et al. 2014). The optical constants of these tholins are taken from Khare et al. (1984). The cloud pressure is 0.001 mbar for the hydrogendominated case and 0.002 mbar for the water-dominated scenario, respectively. Figure 8 shows the resulting transmission spectra for the described scenarios with data from the literature and the radius ratios at different wavelengths obtained by the SOFIA measurements. 

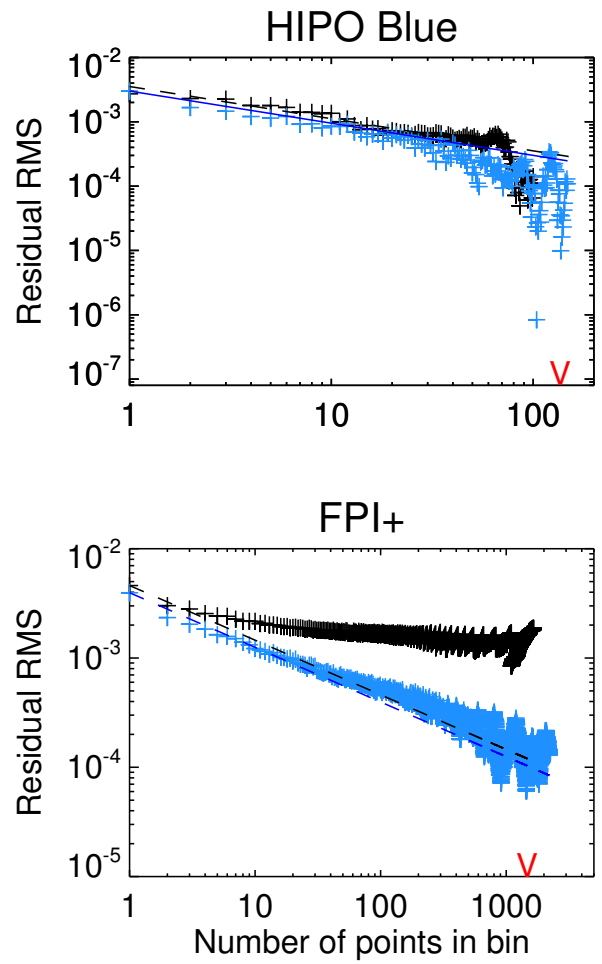
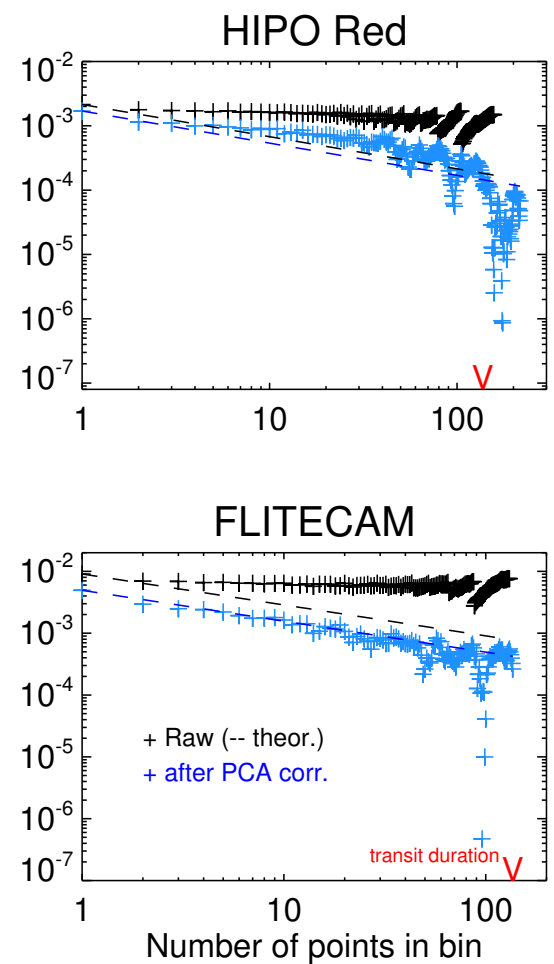

Fig. 6. Residual rms versus bin size for each channel. The black marks represent the values for the raw light curves, whereas the blue corresponds to the de-trended light curves after the best-fit principal component model was subtracted. Dashed lines represent the theoretical values for pure white noise, red arrows mark the transit duration as reference.
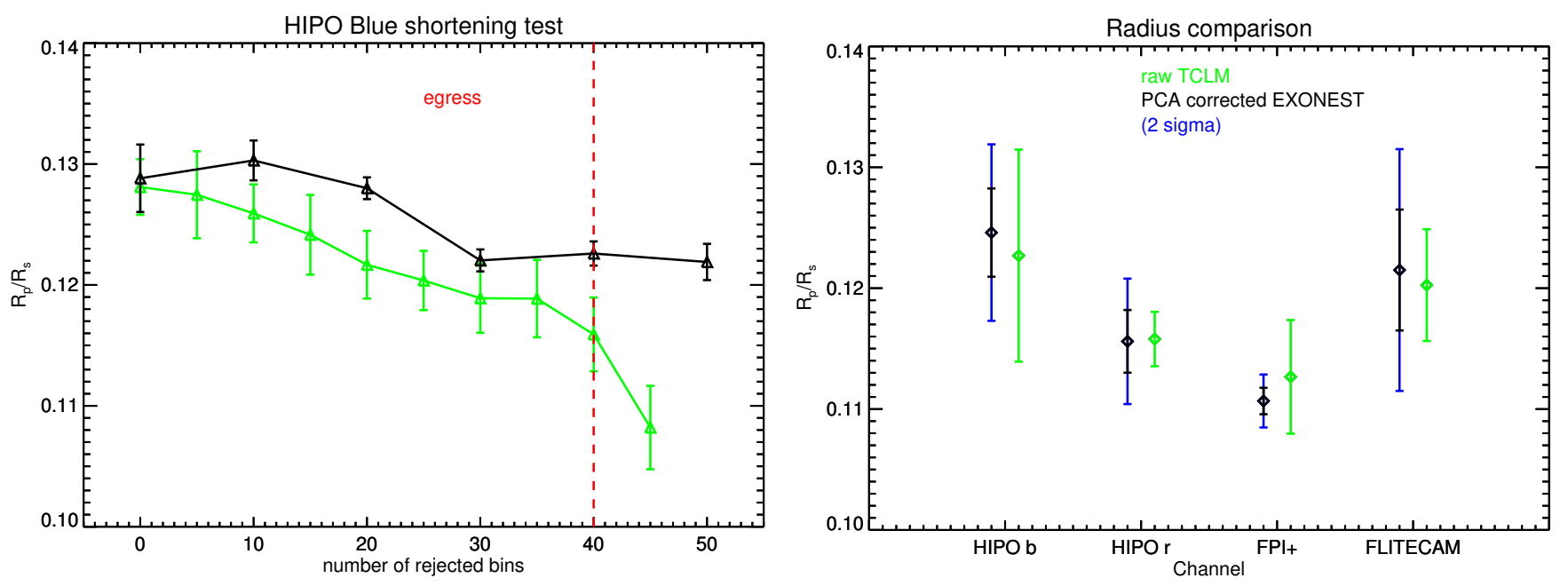

Fig. 7. Left: estimates of the planet-to-star radius ratio for the the HIPO blue observations; from left to right, more data points were neglected from the post-transit observations in steps of 5/10 points. The vertical red line indicates the end of egress, that is, neglecting more points would neglect in-transit data. This indicates that the upward post-transit trend seen in Fig. 4 has affected the measured transit depth. Right: comparison of the results obtained with our two different fitting approaches.

The results clearly suggest that the spread of the obtained photometric data points by SOFIA is larger than the spread in the theoretical transmission spectra. In particular, the very large planetary radius obtained with HIPO blue seems to suggest the presence of a Rayleigh slope, which contradicts the apparent flatness of the planet's spectrum reported by other studies (e.g. Kreidberg et al. 2014). While within the $2 \sigma$ error bars, most photometric points correspond roughly to the simulated spectra. The photometric precision of our SOFIA measurements are unfortunately not good enough to put better constraints on the atmospheric composition than previous studies with similar sensitivities.

\section{Conclusions}

\subsection{Summary and results}

We used FLIPO and FPI+ on board SOFIA to simultaneously observe a transit of the super-Earth GJ 1214b in three optical (openblue $=0.3-0.6 \mu \mathrm{m}, i^{\prime}=0.8 \mu \mathrm{m}$, and $z^{\prime}=0.9 \mu \mathrm{m}$ ) and one never before covered infrared channel (Paschen- $\alpha$ cont. $1.9 \mu \mathrm{m}$ ), and to present the light curves and corresponding transit depths in these bands. We compare to previous observations and current synthetic models of its atmosphere. Unfortunately our results are not sensitive enough to constrain the models any better than previous observations have already done. As discussed in 


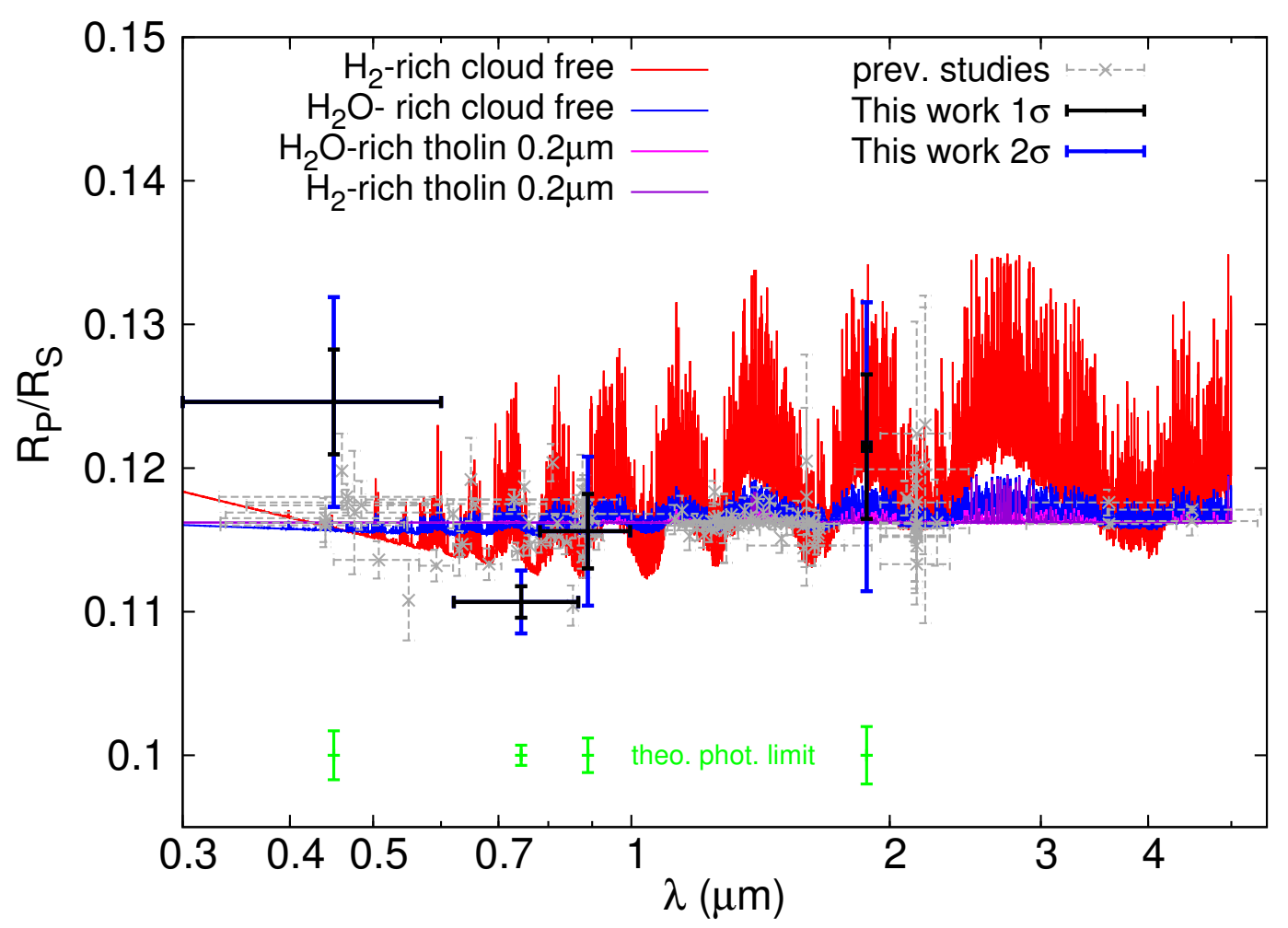

Fig. 8. Model transmission spectra of GJ 1214b, previous measurements (grey) and this work (black, blue). Observations: Bean et al. (2010, 2011); Croll et al. (2011); Crossfield et al. (2011); Désert et al. (2011); Berta et al. (2012); de Mooij et al. (2012); Colón \& Gaidos (2013); de Mooij et al. (2013); Fraine et al. (2013); Cáceres et al. (2014); Kreidberg et al. (2014); Wilson et al. (2014); Nascimbeni et al. (2015); Rackham et al. (2017).

Sects. 3 and 3.3, some of our channels are dominated by residual systematic noise. However, the results could become useful in combination with prior and future observations for future retrievals. We found that the PCA is a powerful tool to reduce the correlated noise in SOFIA data and we recommend its usage for future data analysis. In our present case of GJ 1214b, PCA was able to reduce the noise level by two orders of magnitude and finally we reached a noise level of two times the photon noise. The data also present a second reference for exoplanet transmission spectrophotometry with SOFIA and the first in all four available channels. In Fig. 8 we show the theoretical limits for our observation (green bars). With further improvements to our calibration strategy and a better understanding of our instruments, we are confident that we can get closer to these limits and make SOFIA more competitive in this field. We summarise SOFIA's prospects in the last section.

\subsection{Exoplanets with SOFIA}

In this paper we presented the first exoplanet transit observation with SOFIA that leveraged all four possible channels for simultaneous spectrophotometry. While two of the optical instruments produced good results, the infrared channel did not reach the expected sensitivity. Our measurements suffered from insufficient calibration files for the FLITECAM channel. The challenge is that SOFIA does not have a sufficiently bright flat field source for use with such a narrow band filter as the $1.9 \mu \mathrm{m}$ Paschen$\alpha$ continuum filter. Another lesson learned from this flight is to avoid only short baselines before or after transit at all costs. As the problems with the HIPO blue channel here show, it is crucial to have at least a 30-60 min baseline before and after the occultation to be able to trace systematic changes and correct for instrumental or other observational effects. For future observations we therefore recommend a more careful calibration scheme in particular for obtaining flat fields and if possible a flight plan that allows for more time before and after the transit.

However, even in the current configuration and with all these constrains, there are certain niches that we were able to identify with this and the previous Angerhausen et al. (2015) SOFIA exoplanet observation.

In summary this phase space has the following characteristics:

- bright host stars (like HD 189733b) - for which Angerhausen et al. (2015) demonstrated the ability to perform absolute optical photometry;

- short transit durations;

- science cases that leverage SOFIA's unique capability to observe IR/OPTICAL simultaneously - which complements James Webb Space Telescope (JWST) coverage and can be used for JWST target selection and support;

- transits that are rare or time-critical and require a deployment.

With the upcoming TESS (Transiting Exoplanet Survey Satellite, Sullivan et al. 2015) and PLATO (PLAnetary Transits and Oscillations of stars, Rauer et al. 2014; Hippke \& Angerhausen 2015) missions, we will see a lot more transiting exoplanets that fall into these categories. Furthermore, it is possible to update SOFIA's instrumentation with a modernised optical and infrared precision photometer similar to the previously proposed nearinfrared multi-band ultraprecise spectroimager (NIMBUS) concept (McElwain et al. 2012). This accompanied by a reliable and robust water vapor monitoring system, could make important SOFIA-unique contributions to exoplanet science. 
Acknowledgements. The authors would like to thank the anonymous referee for her or his valuable comments, which substantially helped to improve the quality of the paper. This research has made use of SIMBAD, 2MASS, the GCVS catalogue, and the AAVSO variable search index. D.A. acknowledges the USRA NASA postdoctoral programme and the support of the Center for Space and Habitability of the University of Bern. S.C. acknowledges the Hungarian OTKA Grant K113117. D.K. gratefully acknowledges the support of the Center for Space and Habitability of the University of Bern and the MERAC Foundation for partial financial assistance. This work has been carried out within the frame of the National Centre for Competence in Research PlanetS supported by the Swiss National Science Foundation. D.K. acknowledges the financial support of the SNSF. HIPO work at Lowell is supported by USRA subcontract 8500-98003. FLITECAM work at UCLA is supported by USRA subcontract 08500-05, PI Ian McLean. M.G. acknowledges financial support by the Helmholtz association, PD-015, and the Technische Universität Berlin.

\section{References}

Angerhausen, D. 2013, Exoplanet transits with FLIPO: Is GJ 1214b a water-world Super Earth or a cloudy Mini-Neptune?, SOFIA Proposal, Cycle 2, ID. 02_0053

Angerhausen, D., Krabbe, A., \& Iserlohe, C. 2010, PASP, 122, 1020

Angerhausen, D., Huber, K. F., Mandell, A. M., et al. 2014, in Formation, Detection, and Characterization of Extrasolar Habitable Planets, ed N. Haghighipour, IAU Symp., 293, 435

Angerhausen, D., Mandushev, G., Mandell, A., et al. 2015, J. Astron. Telesc. Instr. Syst., 1, 034002

Barron, J. T., Stumm, C., Hogg, D. W., Lang, D., \& Roweis, S. 2008, AJ, 135, 414

Bean, J. L., Miller-Ricci Kempton, E., \& Homeier, D. 2010, Nature, 468, 669

Bean, J. L., Désert, J.-M., Kabath, P., et al. 2011, ApJ, 743, 92

Benneke, B., \& Seager, S. 2013, ApJ, 778, 153

Berta, Z. K., Charbonneau, D., Bean, J., et al. 2011, ApJ, 736, 12

Berta, Z. K., Charbonneau, D., Désert, J.-M., et al. 2012, ApJ, 747, 35

Bertin, E., \& Arnouts, S. 1996, A\&AS, 117, 393

Brown, T. M., Charbonneau, D., Gilliland, R. L., Noyes, R. W., \& Burrows, A. 2001, ApJ, 552, 699

Cáceres, C., Kabath, P., Hoyer, S., et al. 2014, A\&A, 565, A7

Charbonneau, D., Berta, Z. K., Irwin, J., et al. 2009, Nature, 462, 891

Charnay, B., Meadows, V., Misra, A., Leconte, J., \& Arney, G. 2015, ApJ, 813, L1

Colón, K. D., \& Gaidos, E. 2013, ApJ, 776, 49

Cowan, N. B., Greene, T., Angerhausen, D., et al. 2015, PASP, 127, 311

Cox, A. N. 2000, Allen's Astrophysical Quantities (Springer)

Croll, B., Albert, L., Jayawardhana, R., et al. 2011, ApJ, 736, 78

Crossfield, I. J. M., Barman, T., \& Hansen, B. M. S. 2011, ApJ, 736, 132

Csizmadia, S., Moutou, C., Deleuil, M., et al. 2011, A\&A, 531, A41

Csizmadia, S., Pasternacki, T., \& CEST Team 2013a, in New Quests in Stellar Astrophysics III: A Panchromatic View of Solar-Like Stars, With and Without Planets, eds. M. Chavez, E. Bertone, O. Vega, \& V. De la Luz, ASP Conf. Ser., 472,147

Csizmadia, S., Pasternacki, T., Dreyer, C., et al. 2013b, A\&A, 549, A9

Csizmadia, S., Hatzes, A., Gandolfi, D., et al. 2015, A\&A, 584, A13

Cubillos, P., Harrington, J., Loredo, T. J., et al. 2017, AJ, 153, 3

Czesla, S., Huber, K. F., Wolter, U., Schröter, S., \& Schmitt, J. H. M. M. 2009, A\&A, 505, 1277

de Mooij, E. J. W., Brogi, M., de Kok, R. J., et al. 2012, A\&A, 538, A46

de Mooij, E. J. W., Brogi, M., de Kok, R. J., et al. 2013, ApJ, 771, 109

Désert, J.-M., Bean, J., Miller-Ricci Kempton, E., et al. 2011, ApJ, 731, L40

Dreyer, C. 2013, Transit spectrophotometry of the Super-Earth exoplanet GJ 1214b with FLIPO, SOFIA Proposal, Cycle 2, ID. 02_0084

Dunham, E. W., Elliot, J. L., Bida, T. A., \& Taylor, B. W. 2004, in Groundbased Instrumentation for Astronomy, eds. A. F. M. Moorwood, \& M. Iye, Proc. SPIE, 5492, 592

Dunham, E. W., Elliot, J. L., Brown, T. M., Charbonneau, D., \& McLean, I. S. 2007, in Transiting Extrapolar Planets Workshop, eds. C. Afonso, D. Weldrake, \& T. Henning, ASP Conf. Ser., 366, 256

Dunham, E. W., Bida, T. A., Collins, P. L., et al. 2014, in Ground-based and Airborne Instrumentation for Astronomy V, Proc. SPIE, 9147, 91470H

Eastman, J., Gaudi, B. S., \& Agol, E. 2013, PASP, 125, 83

Espinoza, N., \& Jordán, A. 2015, MNRAS, 450, 1879

Feroz, F., Hobson, M. P., \& Bridges, M. 2009, MNRAS, 398, 1601

Feroz, F., Balan, S. T., \& Hobson, M. P. 2011, MNRAS, 415, 3462

Feroz, F., Hobson, M. P., Cameron, E., \& Pettitt, A. N. 2013, ArXiv e-prints [arXiv: 1306.2144]
Fraine, J. D., Deming, D., Gillon, M., et al. 2013, ApJ, 765, 127 Gaidos, E., Kitzmann, D., \& Heng, K. 2017, MNRAS, 468, 3418 Gazak, J. Z., Johnson, J. A., Tonry, J., et al. 2012, Adv. Astron., 2012, 697967 Geem, Z., Kim, J., \& Loganathan, G. 2001, Simulation, 76, 60

Gehrz, R. D., Angerhausen, D., Becklin, E. E., et al. 2010, in Pathways Towards Habitable Planets, eds. V. Coudé du Foresto, D. M. Gelino, \& I. Ribas, ASP Conf. Ser., 430, 201

Giménez, A. 2006, A\&A, 450, 1231

Grimm, S. L., \& Heng, K. 2015, ApJ, 808, 182

Harpsøe, K. B. W., Hardis, S., Hinse, T. C., et al. 2013, A\&A, 549, A10

Hippke, M., \& Angerhausen, D. 2015, ApJ, 810, 29

Howe, A. R., \& Burrows, A. S. 2012, ApJ, 756, 176

Hu, R., \& Seager, S. 2014, ApJ, 784, 63

Khare, B. N., Sagan, C., Arakawa, E. T., et al. 1984, Icarus, 60, 127

Kreidberg, L., Bean, J. L., Désert, J.-M., et al. 2014, Nature, 505, 69

Logsdon, S. E., McLean, I. S., Becklin, E. E., et al. 2014, in Ground-based and Airborne Instrumentation for Astronomy V, Proc. SPIE, 9147, 91472Y

Mandel, K., \& Agol, E. 2002, ApJ, 580, L171

Marcy, G. W., Weiss, L. M., Petigura, E. A., et al. 2014, PNAS, 111, 12655

McElwain, M. W., Mandell, A., Woodgate, B., et al. 2012, in Ground-based and Airborne Instrumentation for Astronomy IV, Proc. SPIE, 8446, 84467B

McLean, I. S., Smith, E. C., Aliado, T., et al. 2006, in Society of Photo-Optical Instrumentation Engineers (SPIE) Conf. Ser., Proc. SPIE, 6269, 62695B

Menou, K. 2012, ApJ, 744, L16

Metropolis, N., Rosenbluth, A. W., Rosenbluth, M. N., Teller, A. H., \& Teller, E. 1953, J. Chem. Phys., 21, 1087

Miller-Ricci, E., \& Fortney, J. J. 2010, ApJ, 716, L74

Miller-Ricci Kempton, E., Zahnle, K., \& Fortney, J. J. 2012, ApJ, 745, 3

Morley, C. V., Fortney, J. J., Kempton, E. M.-R., et al. 2013, ApJ, 775, 33

Morley, C. V., Fortney, J. J., Marley, M. S., et al. 2015, ApJ, 815, 110

Moutou, C., Hébrard, G., Bouchy, F., et al. 2009, A\&A, 498, L5

Murgas, F., Pallé, E., Cabrera-Lavers, A., et al. 2012, A\&A, 544, A41

Murphy, W. F. 1977, J. Chem. Phys., 67, 5877

Narita, N., Fukui, A., Ikoma, M., et al. 2013a, ApJ, 773, 144

Narita, N., Nagayama, T., Suenaga, T., et al. 2013b, PASJ, 65, 27

Nascimbeni, V., Mallonn, M., Scandariato, G., et al. 2015, A\&A, 579, A113

Nettelmann, N., Fortney, J. J., Kramm, U., \& Redmer, R. 2011, ApJ, 733, 2

Nutzman, P., \& Charbonneau, D. 2008, PASP, 120, 317

Otten, R. H. J. M., \& van Ginneken, L. P. P. P. 1989, The annealing algorithm (Netherlands: Kluwer)

Pál, A. 2008, MNRAS, 390, 281

Pfüller, E., Wiedemann, M., Wolf, J., \& Krabbe, A. 2016, in Ground-based and Airborne Instrumentation for Astronomy VI, Proc. SPIE, 9908, 99082W

Placek, B. 2014, Ph.D. Thesis, State University of New York at Albany

Placek, B., \& Knuth, K. H. 2015, in AIP Conf. Ser., 1641, 447

Placek, B., Knuth, K. H., \& Angerhausen, D. 2014, ApJ, 795, 112

Placek, B., Knuth, K. H., Angerhausen, D., \& Jenkins, J. M. 2015, ApJ, 814, 147

Poddaný, S., Brát, L., \& Pejcha, O. 2010, New Astron., 15, 297

Pont, F., Knutson, H., Gilliland, R. L., Moutou, C., \& Charbonneau, D. 2008, MNRAS, 385, 109

Press, W. H., Teukolsky, S. A., Vetterling, W. T., \& Flannery, B. P. 1992, Numerical recipes (Cambridge University Press)

Rackham, B., Espinoza, N., Apai, D., et al. 2017, ApJ, 834, 151

Rauer, H., Catala, C., Aerts, C., et al. 2014, Exp. Astron., 38, 249

Rogers, L. A., \& Seager, S. 2010, ApJ, 716, 1208

Rothman, L. S., Gordon, I. E., Barber, R. J., et al. 2010, J. Quant. Spectr. Rad. Transf., 111, 2139

Russell, H. N. 1912, ApJ, 35, 315

Sanchis-Ojeda, R., \& Winn, J. N. 2011, ApJ, 743, 61

Silva-Valio, A., \& Lanza, A. F. 2011, A\&A, 529, A36

Sing, D. K., Pont, F., Aigrain, S., et al. 2011, MNRAS, 416, 1443

Sivia, D., \& Skilling, J. 2006, Data analysis: a Bayesian tutorial (USA: Oxford University Press)

Sneep, M., \& Ubachs, W. 2005, J. Quant. Spectr. Rad. Transf., 92, 293

Stetson, P. B. 1987, PASP, 99, 191

Strassmeier, K. G., Granzer, T., Weber, M., et al. 2004, Astron. Nachr., 325, 527

Sullivan, P. W., Winn, J. N., Berta-Thompson, Z. K., et al. 2015, ApJ, 809, 77

Teske, J. K., Turner, J. D., Mueller, M., \& Griffith, C. A. 2013, MNRAS, 431, 1669

Tody, D. 1993, in Astronomical Data Analysis Software and Systems II, eds. R. J. Hanisch, R. J. V. Brissenden, \& J. Barnes, ASP Conf. Ser., 52, 173

Wagner, W., \& Kretzschmar, H.-J. 2008, Internationall Steam Tables: Properties of Water and Steam Based on the Industrial Formulation IAPWS-IF97 (Berlin Heidelberg: Springer-Verlag)

Wilson, P. A., Colón, K. D., Sing, D. K., et al. 2014, MNRAS, 438, 2395 